\title{
Integration Framework for Interoperability of Distributed and Heterogeneous Robot Middlewares
}

\author{
Janarbek Matai, Young-Ho Suh, Hyuongsun Kim, Kang-Woo Lee and Hyun Kim \\ Intelligent Robot Research Division \\ ETRI, 161 Daejon, South Korea \\ \{janarbek, yhsuh,kimhs, kwlee, hkim\}etri.re.kr
}

\begin{abstract}
This paper describes the ongoing development of a software framework for supporting interoperability and integration among heterogeneous network-based robotic development frameworks. We studied two of network based robotic software development frameworks named CAMUS framework and RT-Middleware. The CAMUS(Context-aware Middleware for URC Systems) is a middleware developed by Electronics and Telecommunications Research Institute(ETRI), in Korea, based on the URC(Ubiquitous Robot Companion) concept for developing network based robotic systems. On the other hand, The RT-Middlware was developed by Agency of Industrial Science and Technology(AIST), in Japan, to promote applications of Robot Technology(RT) in various fields. The proposed framework supports interoperability and interconnection among modularized software named RT-Components(RTC) in the context of RTMiddleware and Service Agents(SA) in the context of CAMUS framework. We also implemented and tested some components based on the proposed approach.
\end{abstract}

Index Terms-Networked robotics, Network based robot, interoperability, integration.

\section{INTRODUCTION}

With the proliferation of the communication network technologies, the number of researches and development projects aiming at making robots and robot systems more intelligent by distributing their necessary resources and functionalities over the network are increasing [1], [2], [3], [4]. Recent advances in ubiquitous computing technologies are promoting initiation of various research projects in order to support development of network based robotic systems more easily in a cost effective way. Several attempts have been made to develop frameworks for the development of network based robotic systems such as CAMUS [1], RT-Middleware [6], Player [11], CARMEN [12], ORCA [9], OROCOS [14]. We call this kind of framework a "Network based Robotic Software Development Framework" or simply RSDF.

Though many researches have worked for developing RSDFs, they have some limitations in interoperability between different software systems, because they are inevitably dependent on a specific communication mechanisms, hardware, different operating systems, architectural concepts, propierty source codes, etc. In fact, all RSDFs, mentioned above, have one common goal and one common artifact. The common goal of RSDFs: Enabling software reusability and simplicity. The common artifact of RSDFs: Providing a framework for building reusable, modularized robotic software components(MRSC). In this paper, for the purpose of simplicity,
MRSC is denoted by RSC.

Although, RSDFs has been aiming at enabling software reusability and simplicity, yet, there is an single example for the software reusability and interoperability among different RSDFs. It leads building modularized robotic software components(RSC) that is dependent on a specific RSDF. Moreover, today's RSDFs are developed by different organizations and supported by different communities, which makes it more difficult in supporting interoperability among different RSCs developed by different RSDFs. These kinds of drawbacks in current network based robotic researches limit developing a fully functional, widely accepted network based robotic systems.

Therefore, we proposed the integration framework for supporting interoperability of heterogeneous robot frameworks. It is based on the CAMUS framework which is the reference model of URC (Ubiquitous Robotic Companion) robots.

URC is a new way of developing network based intelligent robots by distributing functional components of robots throughout the network, while keeping a robot's internal scope of abilities at minimal level and providing well-defined interfaces to external resources [1]. The URC concept distributes three functional components of robots such as sensing, acting and processing through the network as in Fig. 1. By distributing main functional elements of the robot through the network, URC reduces the cost of the robot and improves the service qualities. Under the URC concept, ETRI in Korea developed the CAMUS Framework.

On the other hand, the purpose of the RT-Middleware is to modularize robotic functional elements as "Robotic Technology software components" named RT-Components, which would support integrating and extending robotic functioning elements with new functions easily. It empowers building complex robotic systems systematically from the basic elements of the robotic technology. AIST in Japan developed a software named OpenRTM-aist which is a prototype of the RT-Middleware.

In this paper, the integration framework was developed based on the CAMUS framework and the RT-Middleware in order to support the interoperability of heterogeneous network based robotic software development frameworks. The proposed framework empowers several additional advantages: Firstly, it enables software reusability among different network based RSDFs to build complex robotic systems benefiting from their 


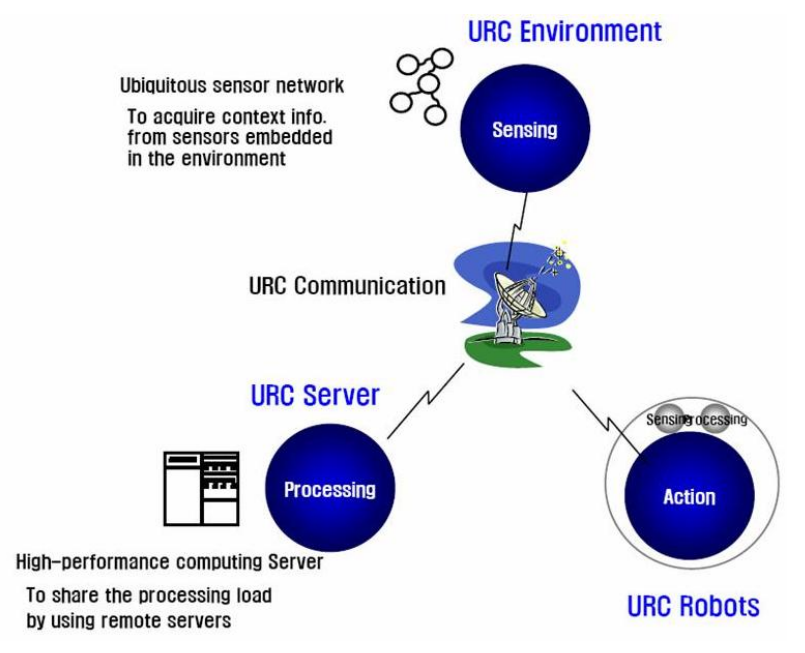

Fig. 1. The URC (Ubiquitous Robot Companion) concept

respective approaches, instead of having to choose only one of them. Secondly, it supports the development of network based robotic systems independently on the modularized software components and communication mechanisms; Finally, it is extensible to different systems. Even though the proposed system is implemented to integrate two systems such as CAMUS framework and RT-Middleware, it can be tailored for the integration of different systems with minimum efforts of changing underlying structures of participating systems.

The reminder of the paper is organized as follows. Section 2 discusses related researches and projects regarding component based robotics software development and its integration. Since the framework is applied to integrate two RSDFs: CAMUS and RT-Middleware, we briefly introduce CAMUS and RTMiddleware in Section 3 and Section 4. Section 5 and Section 6 shows system architecture and implementation in detail. Finally, Section 7 concludes this paper with some remarks and discussion.

\section{RELATED WORK}

Significant amount of researches have been conducted regarding software modularization of network based or conventional robotic systems. Unfortunately, up until now, there is no universally accepted system for the development of network based robots. Most of the works, so far, have devoted to building independent software systems for network based robots. Only few of them stressed the problem of interoperability among those systems. Nevertheless, we have listed some of the significant researches; Player/Stage/Gazebo [11] is and extremely popular robotic software development framework. While Player is a middleware capable of abstracting the control of many different platforms with robot control interfaces, Stage/Gazebo are its simulation backends. Makarenko et al, [9] developed an open-source software project which applies Component-Based Software Engineering principles to the robotic software development. Accoring to [9], software reuse and simplicity is the key continuing progress in robotic

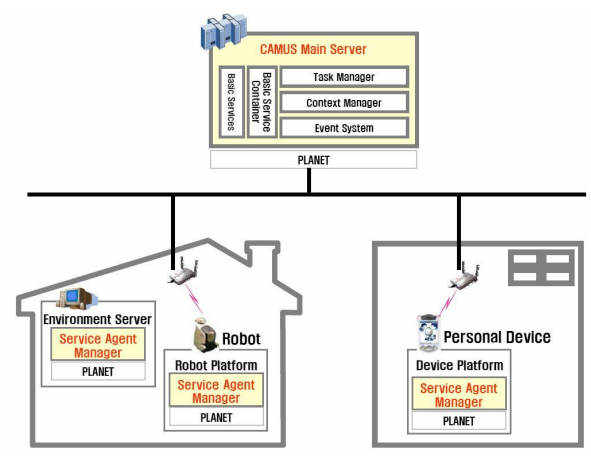

Fig. 2. The CAMUS System organization in physical environment

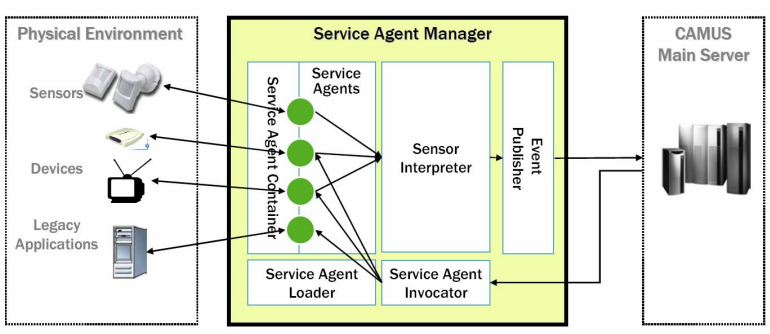

Fig. 3. Service Agent Manager(SAM)

research and industry. Makarenko et al, [10] proposed an approach for reusing modularized software among different robotic software development frameworks. According to [10], 67 percent of current RSCs can be independent of specific RSS, however, yet there is no such independent RSC of specific RSS. Above projects are all tied to specific organization or open-source community. There is no virtually software reuse among current RSSs.

\section{OVERVIEW OF THE CAMUS FRAMEWORK}

CAMUS is a context-aware middleware for the development of network based robotic systems based on the URC concept [1]. It consists of three main sub-systems: Planet, Service Agent Manager(SAM) and CAMUS Main Server. The system organization of CAMUS framework is depicted in Fig. 2.

1) The Planet: Planet provides the light-weight and faulttolerant communication mechanism. It supports the reconnection of disconnected operations as well as asynchronous messaging among various service components. Communication mechanism in CAMUS is carried by the Planet communication protocol.

2) The Service Agent Manager(SAM): The SAM manages and controls sensors, actuators and modularized software components which are called Service Agents(SA). Service Agents may reside on the environment or on the robot platform. SAM acquires environmental information from various sensors and disseminates them to the CAMUS main server. It controls Service Agents in the environment or on the robot platform according to control commands of the CAMUS main server. Fig. 3 shows the architecture of the SAM. 
3) The CAMUS main server: The CAMUS main server integrates information delivered from Service Agent Managers. It consists of several subsystems: Context manager, for controlling and managing context information delivered by sensors; Task manager, for initiating and managing ongoing tasks; Event System, handling events delivered by Context manager and sending them to the Task manager. CAMUS main server also provides the service container to manage service components which have basic functions of robots such as voice recognition, image recognition, text to speech, behavior control, location awareness, and so on.

URC concept specifies well-defined interfaces to abstract the control of many different robot platforms, sensors and actuators. Any robot on the network is able to call and consume URC services, if a robot is registered to the URC services. Current version of CAMUS has all common services needed for any network based robotic software development processes. Speech, Sound, Image, Navigation, and Motion are examples of current available packages for URC service and implemented in CAMUS Framework. Each of this packages defines all necessary interfaces in their own domain. For instance, in the Speech package, TextToSpeech, SpeechRecognizer, and TextToWave inferfaces are included.

\section{OVERVIEW OF THE RT-MidDlEWARE}

RT-Middleware has been built to establish a common platform on the distributed object technology which supports to construct various networked robotic systems by the integration of various network-enabled software components named RT-Components [6] [?]. It provides the following features: The possibility to develop highly scalable component-based systems, The independence of platform and network, The reusability of existing software assets. RT-Middleware consists of :

- A component-based framework

- A set of standard and reusable software modules

- A set of libraries

- A set of standard services

The basic software unit in RT-Middleware is called RTComponent, shortly RTC. RT-Middleware is the environment where RT-Component resides.

RT-Component architecture is shown in Fig. 4.

4) RT-Component: RT-Component can be any modularized software or hardware unit. Cameras, sensors or arms are examples of RT-Components. RT-Components can compose several RT-Components to form a composite RT-Component. For instance, the arm RT-Component consists of manipulator RTComponents. RT-Components are designed and implemented

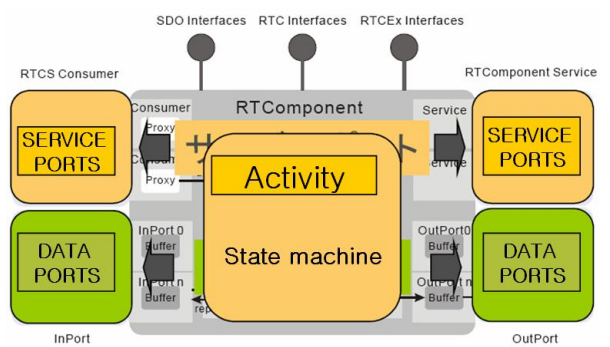

Fig. 4. RT-Component Architecture

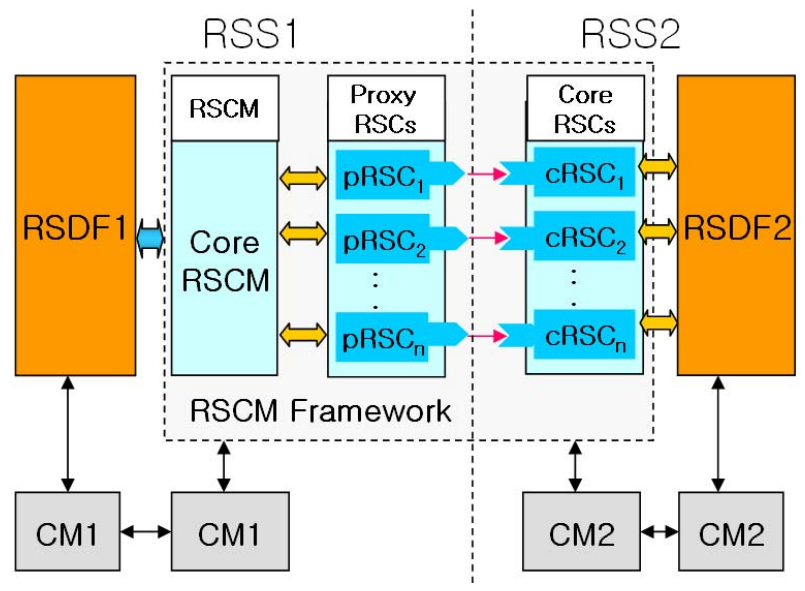

Fig. 5. Overview of the Architecture. RSS1 and RSS2 are two different Robotic Software Systems. Each of them contains different robotic software development frameworks(RSDF1, RSDF2) and different communication middlewares(CM1, CM2).

using distributed object technology so that functionalities provided by different components can be accessed and consumed over the network.

\section{System Architecture}

\section{A. Overview}

The general architecture of the purposed system is depicted in Fig. 5. This architecture supports integration between two different RSDFs such as RSDF1 and RSDF2. Each RSDF was built by different organization based on different communication middleware such as CM1 and CM2, and has different set of RSCs. There are two sets of RSCs such as proxy RSCs and core RSCs. In this architecture, we assume that proxy RSCs(pRSCi where $i=1,2 \ldots n$.) are provided by RSDF1 and core RSCs(cRSCi where $i=1,2 . . n$ ) are provided by RSDF2. In this paper, A RSCM(Robot Software Component Manager) framework was developed for integrating the RSDF1 and the 
RSDF2. Specifically, services provided by modularized robotic softwares components of the RSDF2 are consumed by the RSDF1 by means of proxy RSCs. The RSCM framework supports two-directional interoperability among RSCs of RSDF1 and RSCs of RSDF2. Other words, consuming RSCs of the RSDF1 from the RSDF2 is also possible. The more specific architecture will be discussed in the next section.

This architecture has several advantages; This architecture supports interoperability among different RSCs developed by different RSDFs. On the other hand, any RSDF can coexist with other RSDF on the same robot executing same task having a common goal. Also, other modularized robotic software components provided by other RSDF such as the Player framework [11] can be exchanged with core RSCs easily in this architecture.

\section{B. Architecture of the System}

The proposed architecture was depicted in Fig. 6. The CAMUS and the RT-Middleware framewokrs were utilized in this architecture. (The CAMUS= RSDF1, The RTMiddleware=RSDF2) The CAMUS main server and the RTMiddleware structures' are remained unchanged in this research. The RSCM framework consists of a core RSCM module, Proxy RSCs and Core RSCs.

In this approach, the RSCM serves a role as a middleman between the CAMUS framework and the RT-Middleware via the Planet and the CORBA communication middlewares (CM1=Planet, CM1=CORBA). The Planet communication middleware is used between the CAMUS and the RSCM. The CORBA is used between the RSCM and the RT-Middleware. In this structure, the CAMUS framework will consume core RSCs such as TextToSpeech(TTS), ImageRecognition(IR) and VoiceRecognition(RR) on the RT-Middleware via Proxy RSCs.

1) The core RSCM: The core RSCM is a light-weight middleware which can be deployed on the mobile devices, home robots or on the network based robots. The core RSCM is in charge of managing modularized robotic software components. In this paper, the core RSCM was implemented as a RTComponent which can be executed on the RT-Middleware.

2) Proxy RSCs: Proxy RSCs are the modularized robotic software components provided by the CAMUS framework. It provides interface for the communication between the CAMUS, the core RSCM and core RSCs by proxy RSCs.

3) Core RSCs: Core RSCs are the modularized robotic software components provided by the RT-Middleware. Core RSCs implement the main functionalities of modularized robotic software components which should provide services to the CAMUS main server requests. Proxy RSCs such as pTTS calls core RSC when request arrived from the CAMUS main

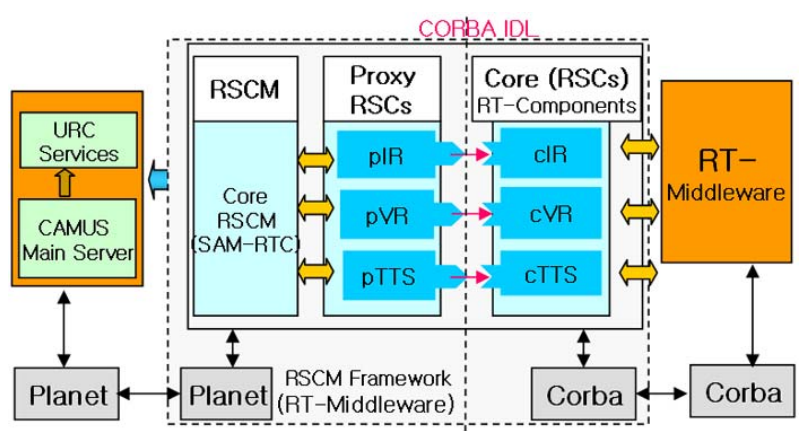

Fig. 6. Architecture of the system based on the CAMUS framework and RTMiddleware. Proxy RSCs are denoted by the prefix $p$ such as pTTS(Proxy TextToSpeech), pIR(Proxy ImageRecognition), pVR(Proxy VoiceRecognition). Core RSCs are denoted by the $c$ prefix such as cTTS(Core TextToSpeech), cIR(Core ImageRecognition), cVR(Core VoiceRecognition)

server. Core RSC are the cornerstone of the RSCM framework since it implements core functionalities of the robotic software in low level. Core RSCs can be in the form of either loadable or executable module.

\section{System IMPLEMENTATION AND EXPERIMENTS}

\section{A. Implementation details}

We applied the suggested approach to three RT-Components of the RT-middleware, which are ImageRecognition, VoiceRecognition and TextToSpeech RT-Components. In this implementation, the client of the CAMUS server consumes services of remote RSCs on the RT-Middleware. The IDL interfaces which are shared between CAMUS server, the RSCM and the provider RT-components are illustrated in Table. I. The flow of the development is as follows.

1) First, the implementation of core the RSCM is done. It is implemented as the SAM in the CAMUS context based on the RT-Middleware. Therefore, we named core RSCM as a SAM-RTC in the implementation.

2) Design of proxy RSCs interfaces based on the URC services.(Proxy RSCs, or simply, Proxy Components)

3) Development of core RSCs based on the RT-Component model on the RT-Middleware.(Core RSCs or Core RTComponents)

4) Development of connector component based on the RTMiddleware which is responsible for connecting proxy RSCs to core RT-Components automatically.

In implementing the system, we consider the structure in Fig. 7. In this structure, we had implemented the RSCM, Proxy components and core RT-Components for robotic functional elements such as Image Recognition, Voice Recognition, Text 


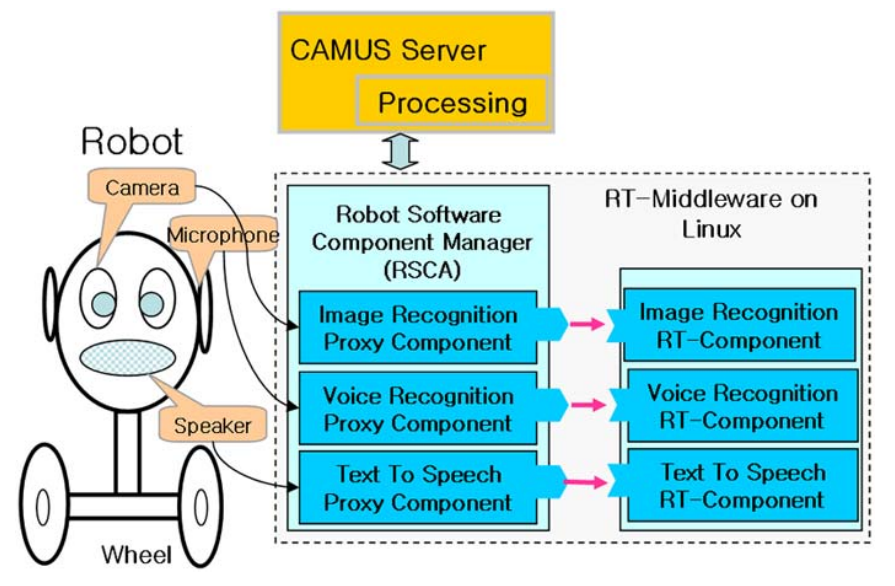

Fig. 7. Structure of the system designed for the experiment

To Speech. We had selected these service because they are important functional elements of both home robots and network based robots.

Core RSCM: The SAM-RTC Implementation: The SAM-RTC was implemented as a service consumer. The SAMRTC consumes services provided by core RT-Components running on the RT-Middleware. It manages core RT-Components by means of Proxy components. The Proxy components and core RT-Components are implemented on the basis of the RT-Middleware command and data exchange feature. Proxy components enable the CAMUS to consume services of core RSCs of other RSDFs.

The SAM-RTC was implemented with following features:

- The SAM-RTC was implemented as a RT-Component.

- The SAM-RTC has three service consumer ports which are called respectively IRPort, TTSPort, and VRPort for consuming services provided by core IR RT-Component, core TTS RT-Component, and core VR RT-Component respectively.

- The SAM-RTC registers consumer ports info to the CAMUS server via the Planet communication protocol enabling requests of the CAMUS Server to Proxy components.

- The SAM-RTC starts/stops the Planet when activating and deactivating the SAM-RTC.

- The SAM-RTC was facilitated with the Planet and the CORBA communication protocols.

Basically, well defined interfaces for common services are shared among the CAMUS server, the SAM-RTC, Proxy components and core RT-Components. All service interfaces are already defined in the URC specification. The CAMUS
TABLE I

TEXTTOSPEECH, VOICERECOGNITION, IMAGERECOGNITION CORBA IDLS FILES. THEY ARE SHARED AMONG THE CAMUS, CORE RSCM AND CORE RSCS.

\begin{tabular}{|l|l|}
\hline Interface names: & Interfaces methods \\
\hline TextToSpeech: & $\begin{array}{l}\text { void speak(String text) } \\
\text { char* getAvailableVoices() } \\
\text { void cancelAll(String details) }\end{array}$ \\
\hline VoiceRecognition: & $\begin{array}{l}\text { void play(InputStream is) } \\
\text { void playBytes(byte[] wave) } \\
\text { voidplayFile(String path) } \\
\text { void cancelAll(String details) }\end{array}$ \\
\hline ImageRecognition: & $\begin{array}{l}\text { void openCamera() } \\
\text { void captureImage() } \\
\text { void getImageFormat() }\end{array}$ \\
\hline
\end{tabular}

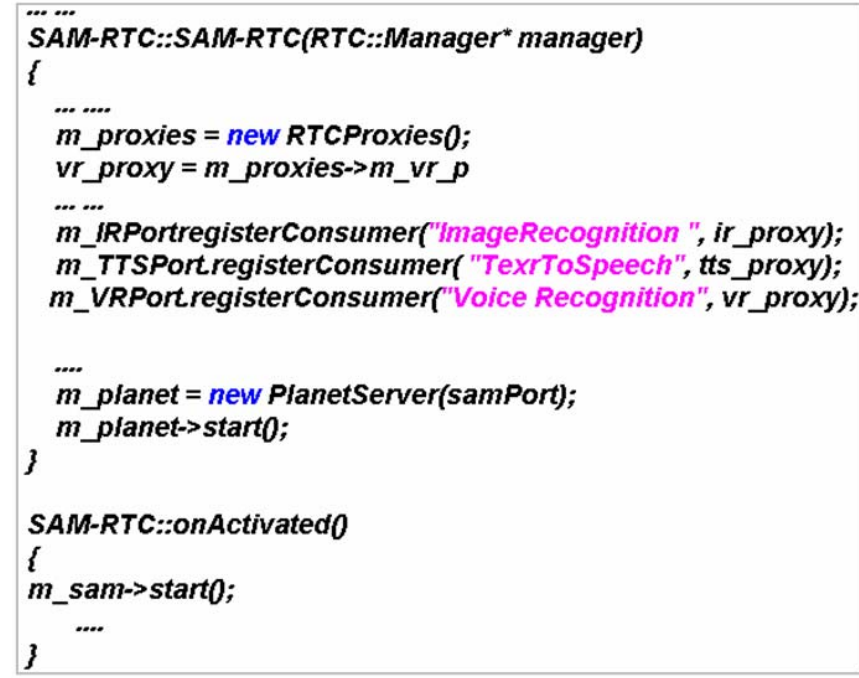

Fig. 8. An example source code for SAM-RTC class

framework includes all basic services for developing network based robots. Interface files for TextToSpeech, Voice Recognition, Image Recognition are depicted in Table. I. At starting point of the SAM-RTC, it registers Proxy components to the CAMUS main server and to the CORBA naming service in the RT-Middleware. From that point the CAMUS can access any service provided by core RT-Components in the RTMiddleware such as core TTS, core IR and core VR. An example source code for the SAM-RTC is depicted in Fig. 8.

Design of proxy RSCs: All proxy RSCs or proxy components inherit from Urc.services package. Proxy RSCs enable the CAMUS to consume services of core RT-Components on the RT-Middleware. Urc.services provides all necessary classes for developing basic services in the CAMUS frame- 


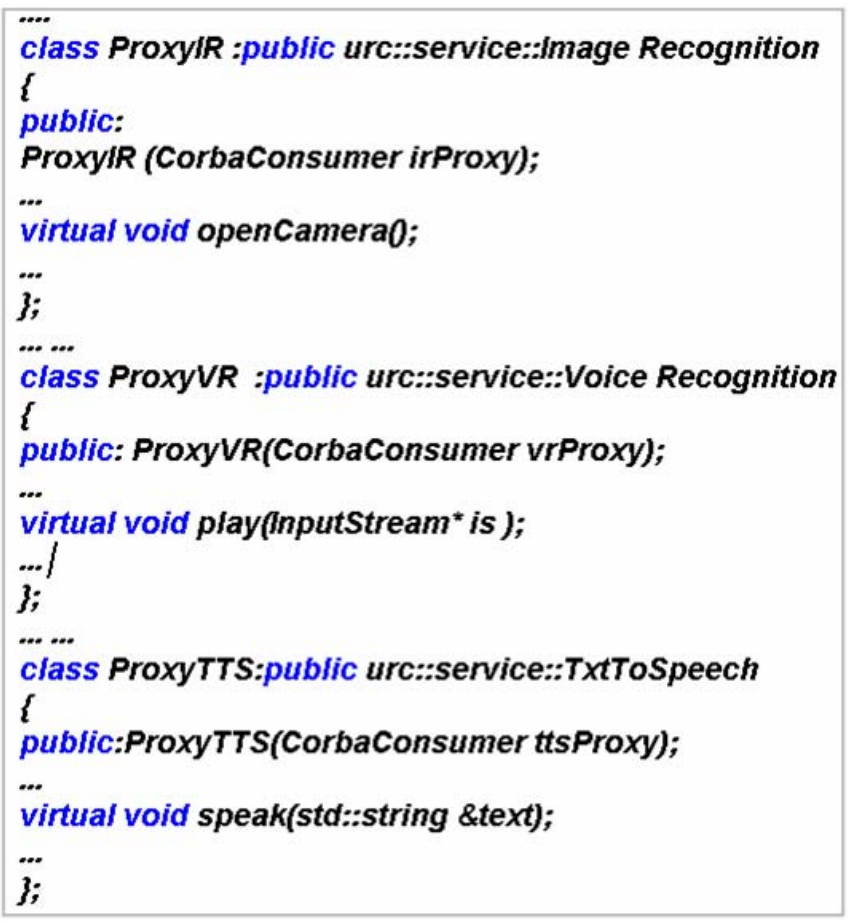

Fig. 9. An example source code for Proxy Components pIR, pVR and pTTS

TxtToSpeech *provider = new TxtToSpeech $\left(m_{-}\right.$tts_p $)$; servant=new urc::service::TxtToSpeech ( $m$ _planet, provider $)$; loadService("TxtToSpeech",servant);

Fig. 10. An example source for registering Proxy pTTS to the CAMUS main server

work. ImageRecognition, TextToSpeech, VoiceRecognition proxies RSCs are inherited from corresponding services in URC.service and implemented in the SAM-RTC. Fig. 9 shows proxy RSCs for the TextToSpeech, the VoiceRecognition and the ImageRecognition.

Proxy RSCs should be registered to the CAMUS main server at the starting point of the SAM-RTC. Fig. 10 lists an example source code for registering the TextToSpeech proxy $\mathrm{RSC}$ to the CAMUS main server.

Core RT-Component implementation: The development process of core RT-Components are straightforward. In this experiment, TextToSpeech, VoiceRecognition and ImageRecognition core RT-Components are implemented based on the consumer/provider feature of the RT-Middleware according to shared interfaces in Table I.

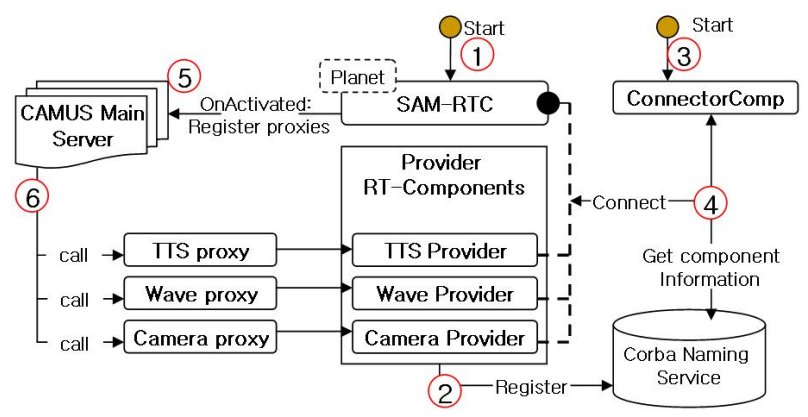

Fig. 11. Sequence of operations for the experiment.

Connector Component: Finally, a connector component is implemented for connecting SAM-RTC's consumer ports to corresponding core RT-Components' provider ports. The connector component has several roles; it queries the CORBA naming service and finds available components, connects newly added core RT-Components' provider port to the SAMRTC's consumer port.

\section{B. Experiment Results}

1) System resources: We have utilized three servers. the CAMUS main server was running on Windows XP Professional (Pentium $2.5 \mathrm{GHz}$ ), and the TTS server was running on Linux with Pentium 4, $2.4 \mathrm{GHz}$. The SAM-RTC, VoiceRecognition, ImageRecognition RT-Components were implemented on the OpenRTM-aist 0.4 version under the linux Fedora core 4. The ImageRecognition component was implemented using the OpenCV library from IBM on Windows XP Professional with Pentium $2.5 \mathrm{GHz}$. LogiTech QuichCam was utilized by the core ImageRecognition RT-Component. Other software package used for the system were omniORB 4.0(CORBA), ACE 5.5, OpenCV 1.0, Iconv.

2) Experiment: The sequence of operations involved in this experiment is shown in Fig. 12. The flow of the communication model is as follows.

1) The RTC Manager initializes IR, VR and TTS RTComponents and registers them to the CORBA naming services as in Fig. 13. The initiated components can be seen from the RTCLink tool which is provided by RTMiddleware. as in Fig. 13. (Step (1) and (2))

2) Then connector component starts and starts to queries the CORBA naming service and connects RSCM's consumer ports to corresponding ports of core RTComponents. (Step (3) and (4))

3 ) If component states are changed from the inactive state to the active state, the RSCM starts the Planet commu- 


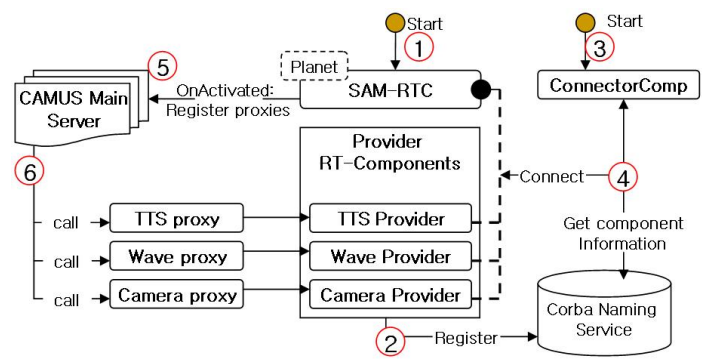

Fig. 12. Sequence of operations for the experiment.

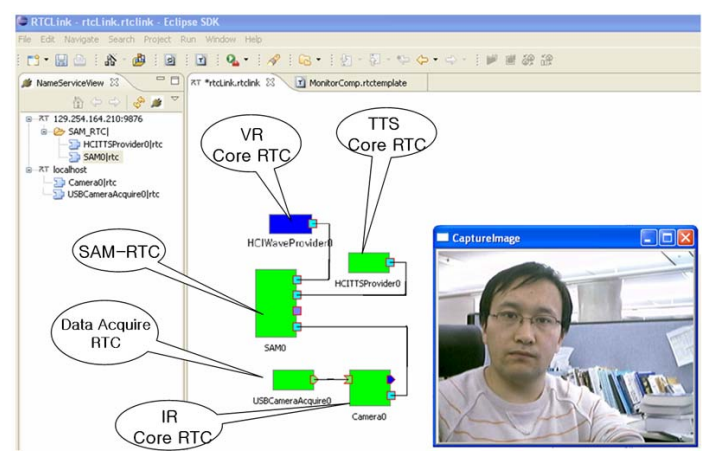

Fig. 13. The RTCLink tool provided by the RT-Middleware. Currently, core TTS, IR RT-Components are actively running on the RT-Middleware. Core VR RT-Component is in the inactive state.

nication middleware and registers Proxy objects to the CAMUS Server. (Step (5))

4) Finally, our core RT-Components are ready to respond to the requests from the CAMUS main server as in the Fig. 13. (Step (6))

From this point, the CAMUS can request the ImageRecognition RT-Component to open a USB Camera or send a text file to the core TextToSpeech RT-Component on the RT-Middleware to convert text to speech. In Fig. 13 the core ImageRecognition RT-Component was responded to the CAMUS main server request opening the USB Camera(LogiTech QuichCam), and it started sending captured image to the CAMUS main server.

\section{CONCLUSION AND DISCUSSION}

In this paper, we discussed the system integration framework for network based robots by integrating two well-known network based robot software development frameworks: the CAMUS Framework and RT-Middleware. Basically, the interoperability of RSCs(Service Agents) of the CAMUS and RSCs(RT-Componets) of RT-Middleware was discussed and implemented for several cases: A TextToSpeech, a VoiceRe- cognition, and an ImageRecognition RT-Components were implemented and consumed with the CAMUS main server;

We believe that interoperability of robotic software components of different RSDFs are important for following reasons; It supports software reuse among different robotic software development frameworks on the distributed environment, avoids overlapping implementation of same kind of software, more choice for robot builders, and promotes systematic software use in robotics application.

In addition, the proposed framework augments integration of heterogeneous distributed network based robotic software frameworks easily and supports interoperability among modularized software components of different frameworks. By doing so, the current frameworks will remain same, while robotic software developers would have more choice in terms of cost and ease of use of modularized robotic software components.

\section{REFERENCES}

[1] Hyun Kim, Minkyoung Kim, Kang-Woo Lee, Young-Ho Suh, Joonmyun Cho, Young-go Cho,Context-Aware Server Framework for Network-based Service Robots, SICE-ICASE, Oct. 18-21, 2006, Page(s):113- 120.

[2] Hyun Kim Young-Jo Cho Sang-Rok Oh, the CAMUS: a middleware supporting context-aware services for network based robots, Advanced Robotics and its Social Impacts, IEEE Workshop, Jun.12-15, 2005, Page(s):237- 242.

[3] Young-Guk Ha, Joo-Chan Sohn, Young-Jo Cho, and Hyunsoo Yoon ,Towards a Ubiquitous Robotic Companion:Design and Implementation of Ubiquuitous Robotic service Framework, ETRI Journal, Dec, 2006, Vol:27, Page(s):666- 676.

[4] Aekyoung Moon, Hyongson Kim, Kangwoo Lee, and Hyun Kim ,Designing CAMUS based Context-Awareness for Prvasive Home Environements, ICHIT, Nov.9-11, 2006, Cheju Island, S.Korea.

[5] RT-Middleware, http://www.is.aist.go.jp/rt/OpenRTM-aist/html-en/

[6] Ando, N. Suehiro, T. Kitagaki, K. Kotoku, T. Woo-Keun Yoon , RTmiddleware: distributed component middleware for RT (robot technology), IROS,2-6 Aug. 2005

[7] Carle Cote, Yannick Brosseau, Dominic Letourneau, Clement Raievsky, Francois MichaudY, Robotic Software Integration Usign Marie, Journal of Advanced Robotic Systems, Vol.3, No.1 (2006), Page(s):55- 60.

[8] Anthony Mallet, Fumio Kanehiro, Sara Fleury and Matthieu Herrb, Reusable Robotics Software Collection,

[9] A. Makarenko, A. Brooks, T. Kaupp. Orca: Components for Robotics. IROS 2006. Workshop on Robotic Standardization.

[10] A. Makarenko, A. Brooks, T. Kaupp, On the Benefits of Making Robotic Software Framework Thin. IROS 2007.

[11] Brian Gerkey, Richard T. Vaughan and Andrew Howard. "The Player/Stage Project: Tools for Multi-Robot and Distributed Sensor Systems". (ICAR 2003), pages 317-323, Coimbra, Portugal, June 2003.

[12] Sebastian Thrun, Dieter Fox, Wolfram Burgard, and Frank Dellaert, Robust Monte Carlo Localization for Mobile Robots,Journal of Artificial Intelligence, Vol.128, 2000.

[13] Makoto MIZUKAWA, ORiN: Standard Network Interface for Robot/Factory Automation, IROS Workshop on Robot Middleware and Standardization, 2004, Sendai, Japan

[14] http://www.orocos.org/

[15] High Performance ORB http://omniorb.sourceforge.net/

[16] http://robotics.omg.org/

[17] Common Object Request Broker Architecture, http://www.corba.org/ 